\title{
Fertilização com N e S na Recuperação de Pastagem de Brachiaria brizantha cv. Marandu em Neossolo Quartzarênico ${ }^{1}$
}

\section{Patrícia Perondi Anchão Oliveira², Paulo Cesar Ocheuze Trivelin ${ }^{3}$, Wladecir Salles de Oliveira ${ }^{4}$, Moacyr Corsi ${ }^{5}$}

\begin{abstract}
RESUMO - Com o objetivo de avaliar a resposta de pastagens degradadas de Brachiaria brizantha cv. Marandu ao uso de diferentes doses de fertilização com $\mathrm{N}$ e S, foi desenvolvido um experimento em campo durante dois anos, em área de cerrado em blocos ao acaso. Foram avaliados sete tratamentos, seis com diferentes combinações de doses de $\mathrm{N}$ e $\mathrm{S}$ e uma testemunha (pastagem degradada). As combinações foram: $70 \mathrm{~kg} \mathrm{ha}^{-1} \mathrm{~N}$ sem enxofre, $140 \mathrm{~kg} \mathrm{ha}^{-1} \mathrm{~N}$ sem enxofre, $210 \mathrm{~kg} \mathrm{ha}^{-1} \mathrm{~N}$ sem enxofre, $70 \mathrm{~kg} \mathrm{ha} \mathrm{N}^{-1} \mathrm{~N}$ e $77 \mathrm{~kg} \mathrm{ha}{ }^{-1} \mathrm{~S}, 140$ $\mathrm{kg} \mathrm{ha}^{-1} \mathrm{~N}$ e $153 \mathrm{~kg} \mathrm{ha}^{-1} \mathrm{~S}, 210 \mathrm{~kg} \mathrm{ha}^{-1} \mathrm{~N}$ e $230 \mathrm{~kg} \mathrm{ha}^{-1} \mathrm{~S}$. A reposta em produção de forragem ao uso de fertilização nitrogenada foi linear, com maior eficiência de uso do nitrogênio no segundo ano de manejo. A maior produção de forragem foi acompanhada por maior extração de $\mathrm{N}$ e queda nos teores de matéria orgânica no solo. A extração de enxofre foi menor que as doses de $\mathrm{S}$ empregadas. O critério da relação $\mathrm{N}$ :S na forragem na nutrição mineral da forrageira foi recomendado apenas na época das águas. Houve acúmulo de enxofre nas camadas subsuperficiais do solo.
\end{abstract}

Palavras-chave: degradação, fertilizantes, uréia, sulfato de amônio, relação N:S, nutrientes

\section{$\mathrm{N}$ and S Fertilization and Recovery of Brachiaria brizantha cv. Marandu Pasture on Sandy Soil}

\begin{abstract}
An experiment was carried out during two years on Cerrado soil with the objective of evaluating the response of degraded Brachiaria brizantha cv. Marandu pastures to different N and S fertilization doses. Seven treatments, six with different $\mathrm{N}$ and $\mathrm{S}$ combinations and a control (degraded pasture), were compared in a randomized complete blocks design. The combinations were: 70

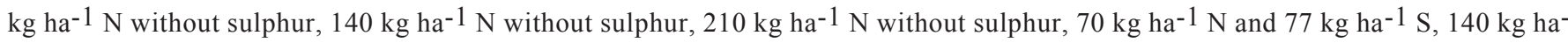
${ }^{1} \mathrm{~N}$ and $153 \mathrm{~kg} \mathrm{ha}^{-1} \mathrm{~S}, 210 \mathrm{~kg} \mathrm{ha}^{-1} \mathrm{~N}$ and $230 \mathrm{~kg} \mathrm{ha}^{-1} \mathrm{~S}$. Herbage yield response to nitrogen rates was linear, and the best efficiency of nitrogen use was obtained in the second year. The highest herbage yield was associated with higher extraction of $\mathrm{N}$ and smaller soil organic matter content. The sulfur extraction was lower than $\mathrm{S}$ doses. The N:S ratio as a criterion of the forage mineral nutrition was effective only in the rainy season. There was sulphur accumulation in deeper layers of soil.
\end{abstract}

Key Words: degradation, fertilizer, urea, ammonium sulfate, ratio $\mathrm{N}: \mathrm{S}$, nutrients

\section{Introdução}

A degradação de pastagens é um dos tópicos mais relevantes da pecuária nacional. Estima-se que aproximadamente $30 \%$ dos 160 milhões de hectares de pastagens do Brasil estejam degradados (Zimmer et al., 1994). A recuperação destas áreas é fundamental em termos econômicos, técnicos e ambientais.

Para a recuperação destas áreas, são fundamentais a melhoria da fertilidade do solo e o manejo adequado da planta forrageira (Anchão, 1997). O manejo da fertilidade do solo em áreas de pastagens degradadas difere do realizado em áreas recém- implantadas ou manejadas intensivamente há muitos anos. A resposta ao uso de fertilizantes em pastos degradados aumenta durante o processo de recuperação (Oliveira et al., 2003).

O acúmulo de nutrientes na parte aérea de pastagens em processo de recuperação é alto. Oliveira et al. (2000) encontraram acúmulo e posterior exportação de 326, 38 e $22 \mathrm{~kg} \mathrm{ha}^{-1} \mathrm{ano}^{-1}$ de N, P e S, respectivamente, durante a recuperação de uma pastagem de Brachiaria brizantha cv. Marandu. Mesmo sendo baixa a exportação de nutrientes pelos animais destinados à produção de carne, leite e lã, a alta extração pelas plantas, somada às perdas e à

\footnotetext{
1 Projeto financiado pelo SN - Centro de Pesquisa e Promoção do Sulfato de Amônio.

2 Pesquisadora da EMBRAPA - Centro de Pesquisa de Pecuária do Sudeste, Rodovia Washington Luiz, km 234 , Caixa Postal 339 - São Carlos, SP - CEP: 13560-970. E.mail: ppaolive@cppse.embrapa.br

${ }^{3}$ Professor do Laboratório de Isótopos Estáveis do CENA - USP, Piracicaba, SP. Bolsista do CNPq. E.mail: pcotrive@cena.usp.br

${ }^{4}$ Engenheiro Agrônomo, Dr., Microquímica Ind. Químicas Ltda., Campinas, SP. E.mail: wsolivei@gmail.usp.br

5 Professor do Departamento de Zootecnia da ESALQ - USP, Piracicaba, SP. E.mail: moa@esalq.usp.br
} 
ineficiência da reciclagem de nutrientes das dejeções animais, principalmente do nitrogênio (Corsi \& Martha Jr., 1997; Boddey et al., 2000), comprometem a sustentabilidade das pastagens, levando à degradação.

Neste contexto, entre todos os nutrientes, o nitrogênio tem merecido bastante atenção, em razão dos seguintes motivos: reciclagem no sistema comprometida pela distribuição desuniforme e pelas altas perdas ocorridas nas dejeções dos animais (Corsi \& Martha Jr., 1997); esgotamento do nitrogênio do sistema levando à degradação das pastagens (Boddey et al., 2000; Corsi \& Martha Jr., 1997); elevado requerimento em quantidade e maior custo desse nutriente, essencial para a exploração de sistemas intensivos de pastagens (Stout, 1995). Outras perdas, como as gasosas (desnitrificação e volatilização de amônia), a lixiviação e os custos envolvidos durante a fertilização nitrogenada, também concorrem, elevando a importância dos fenômenos envolvidos com o uso de $\mathrm{N}$ em pastagens (Oliveira, 2001).

Outro fato que pode interferir na resposta da planta forrageira à adubação nitrogenada é a fertilização com enxofre, uma vez que existe uma estreita relação entre esses dois nutrientes que interfere tanto na produção quanto na qualidade da planta forrageira. Como o enxofre participa da formação de dois aminoácidos essenciais (a cistina e a metionina), sua deficiência interrompe a síntese de proteínas (NRC, 1989). Em plantas deficientes em enxofre, o crescimento é retardado (Raij, 1991), mesmo havendo suprimento adequado de outros nutrientes, como o nitrogênio. Existem relatos de que a deficiência de enxofre atinge cerca de $50 \%$ da área total dos solos da América Tropical (Vitti \& Novaes, 1986).

A extração de enxofre pelas plantas forrageiras pode ser alta, em torno de $50 \mathrm{~kg} \mathrm{ha}^{-1} \mathrm{ano}^{-1}$, considerando-se produtividade de $20 \mathrm{t} \mathrm{ha}^{-1} \mathrm{ano}^{-1}$ de MS e concentração de $\mathrm{S}$ na parte aérea de $2,5 \mathrm{~g} \mathrm{~kg}^{-1}$ (Werner et al., 1996). O conhecimento da dose de enxofre adequada para atender as necessidades da planta forrageira é importante não só pela magnitude da extração como também pela possível perda do nutriente por lixiviação, que é favorecida pela correção do solo, pela fosfatagem e pela irrigação (Nguyen \& Goh, 1994). Santos \& Monteiro (1999), em condições controladas, obtiveram aumento na produção de MS e no número de perfilhos de Brachiaria brizantha cv. Marandu, em função de diferentes doses de enxofre, o que denota a importância da nutrição mineral adequada de enxofre para plantas forrageiras. Além da quantidade de enxofre, também é importante na produção de forragem a relação $\mathrm{N}: \mathrm{S}$, em virtude das dificuldades para se avaliar o teor crítico de $\mathrm{S}$ para forrageiras, uma vez que o conteúdo do nutriente reduz com o crescimento da planta, enquanto a relação $\mathrm{N}: \mathrm{S}$ permanece constante nos diversos estádios da planta forrageira (Vitti \& Novaes, 1986). Relações em torno de 16,5:1 são adequadas para garantir o desenvolvimento adequado de plantas forrageiras (Scott, 1983).

$\mathrm{O}$ uso de fertilizantes concentrados em N, P e K nas adubações em pastagens não fornecem $\mathrm{S}$ ao sistema. A substituição de fontes concentradas de fertilizantes, como a uréia, por outras, como o sulfato de amônio, pode ser interessante no processo de recuperação de pastagens por fornecer $\mathrm{S}$.

Com o objetivo de avaliar a resposta de pastagens degradadas à fertilização nitrogenada e sulfatada, foi desenvolvido um experimento em que se avaliaram três doses de nitrogênio na presença ou ausência de fertilização com enxofre durante dois anos.

\section{Material e Métodos}

O experimento foi realizado na Fazenda Mercedes,

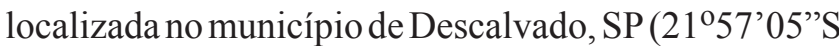
e 47³ '26”W) com altitude de $679 \mathrm{~m}$. A área experimental possuía pastagem degradada de Brachiaria brizantha cv. Marandu estabelecida há apenas dois anos em Neossolo Quartzarênico, com pH em $\mathrm{CaCl}_{2}=$ 4,0 ; matéria orgânica $=20 \mathrm{~g} \mathrm{dm}^{-3} ; \mathrm{P}=1 \mathrm{mg} \mathrm{dm}^{-3}$; e 0,$8 ; 2 ; 1 ; 12 ; 54 \mathrm{mmol}_{\mathrm{c}} \mathrm{dm}^{-3}$ de $\mathrm{K}, \mathrm{Ca}, \mathrm{Mg}, \mathrm{Al} \mathrm{e} \mathrm{CTC}$, respectivamente; saturação por bases de $7 \%$; saturação por alumínio de $76 \%$ e S $\left(\mathrm{Ca}\left(\mathrm{H}_{2} \mathrm{PO}_{4}\right)_{2}\right)=1 \mathrm{mg} \mathrm{dm}^{-3}$; conforme Raij et al. (1987).

O delineamento experimental foi o de blocos casualizados, com seis tratamentos compostos por diferentes combinações de $\mathrm{N}$ e $\mathrm{S}$ e quatro repetições e um tratamento testemunha sem uso de correção ou fertilização do solo (Tabela 1).

As parcelas, medindo $2 \times 5 \mathrm{~m}$, foram instaladas no campo em julho de 1998, realizando-se calagem com 4,5 toneladas ha-1 de calcário dolomítico PRNT 90. Em novembro de 1998, foi realizado um corte de uniformização a $0,20 \mathrm{~m}$ de altura e iniciaram-se as fertilizações. Foram aplicados a lanço na superfície do solo $80 \mathrm{~kg}$.ha-1 de $\mathrm{P}_{2} \mathrm{O}_{5}$ na forma de termofosfato magnesiano, $30 \mathrm{~kg} \mathrm{ha}^{-1}$ de FTE BR 12 e cloreto de potássio suficiente para igualar a dose de K em $274 \mathrm{~kg} \mathrm{ha}^{-1}$ de $\mathrm{K}_{2} \mathrm{O}$ entre os tratamentos, para atingir $5 \%$ de $\mathrm{K}$ na CTC, utilizando-se eficiência de 70\%. As fertilizações de 
Tabela 1 - Tratamentos

Table 1 - Treatments

\begin{tabular}{lcc}
\hline $\begin{array}{l}\text { Tratamento }\left(\mathrm{kg} \mathrm{ha}^{-1} \mathrm{ano}^{-1}\right) \\
\text { Treatment }\left(\mathrm{kg} \mathrm{ha}^{-1} \text { year }\right.\end{array}$ & $\begin{array}{c}\text { Fonte de nitrogênio } \\
\text { Nitrogen source }\end{array}$ & $\begin{array}{c}\text { Fórmula } \\
\text { formula }\end{array}$ \\
\hline $\begin{array}{l}\text { Testemunha } \\
\text { Control }\end{array}$ & \multicolumn{2}{c}{$\begin{array}{c}\text { Sem correção do solo e fertilização } \\
\text { Without liming and fertilization }\end{array}$} \\
$70 \mathrm{~kg} \mathrm{~N}$ & Uréia (urea) & 25.00 .25 \\
$140 \mathrm{~kg} \mathrm{~N}$ & Uréia (urea) & 25.00 .25 \\
$210 \mathrm{~kg} \mathrm{~N}$ & Uréia (urea) & 25.00 .25 \\
$70 \mathrm{~kg} \mathrm{~N} 77 \mathrm{~kg} \mathrm{~S}$ & Sulfato de amônio (ammonium sulfate) & 15.00 .15 \\
$140 \mathrm{~kg} \mathrm{~N} \mathrm{e} 153 \mathrm{~kg} \mathrm{~S}$ & Sulfato de amônio (ammonium sulfate) & 15.00 .15 \\
$210 \mathrm{~kg} \mathrm{Ne} 230 \mathrm{~kg} \mathrm{~S}$ & Sulfato de amônio (ammonium sulfate) & 15.00 .15 \\
\hline
\end{tabular}

cobertura ocorreram após cada corte da estação chuvosa durante os dois anos experimentais (entre os meses de novembro e março de cada ano), aplicando-se $70 \mathrm{~kg} \mathrm{ha}^{-1}$ de $\mathrm{N}, 70 \mathrm{~kg} \mathrm{ha}^{-1}$ de $\mathrm{K}_{2} \mathrm{O}$ e 77 $\mathrm{kg} \mathrm{ha}^{-1}$ de $\mathrm{S}$ em cada cobertura. O número de coberturas definiu as diferentes doses de $\mathrm{N}$ e $\mathrm{S}$ dos tratamentos. Assim, os tratamentos com doses de $70 \mathrm{~kg} \mathrm{ha}^{-1}$ de $\mathrm{N}$ e $\mathrm{S}$ receberam uma única cobertura no mês de novembro e os tratamentos com $140 \mathrm{~kg} \mathrm{ha}^{-1}$ de $\mathrm{N}$ e S, duas coberturas, uma em novembro e outra em janeiro, e finalmente os tratamentos que receberam $210 \mathrm{~kg} \mathrm{ha}^{-1}$ de $\mathrm{N}$ e $\mathrm{S}$ receberam três coberturas (em novembro, janeiro e fevereiro). Os tratamentos que não continham $\mathrm{S}$ receberam uréia como fonte de $\mathrm{N}$ e os que continham $\mathrm{S}$, sulfato de amônio.

Em julho de 1999, realizou-se novamente a calagem para atingir saturação por bases de $80 \%$, de acordo com os resultados médios obtidos na análise de solo para cada tratamento ao fim do primeiro ano. Em novembro de 1999 , realizaram-se novamente as fertilizações de correção, com $80 \mathrm{~kg} \mathrm{ha}^{-1}$ de $\mathrm{P}_{2} \mathrm{O}_{5}$ na forma de termofosfato magnesiano e com $\mathrm{K}$, para atingir 5\% de $\mathrm{K}$ na $\mathrm{CTC}$, conforme requerimento de cada tratamento e prosseguiu-se com as fertilizações de cobertura, como já especificado.

Os cortes da estação chuvosa foram realizados a cada 35 dias e da estação seca, a cada 60 dias, conforme avaliação do crescimento e da senescência da planta forrageira, somando-se seis cortes da forrageira no ano agrostológico de 1998/1999 e sete cortes em 1999/2000. A altura de corte foi de $20 \mathrm{~cm}$. Em outubro de cada ano, foi avaliado o resíduo de forragem existente na área. Foram avaliadas as produções de matéria seca de raízes, por meio de amostragem com sonda, separando-se por meio de lavagem em peneira de 20 mesh as raízes do solo, e de matéria seca da parte aérea, por meio do corte manual de quatro subamostras de 0,3 x 0,6 m, tomadas ao acaso em cada parcela. As amostras foram secas a $65^{\circ} \mathrm{C}$ até atingirem peso constante. O acompanhamento da fertilidade de solo foi realizado nas profundidades de 0-0,05, 0,05-0,10 e 0,10-0,20 m, em julho de 1999 e em dezembro de 2000. Foram realizadas determinações dos cátions trocáveis $(\mathrm{Ca}, \mathrm{Mg}, \mathrm{K}, \mathrm{H}$ e Al) e de $\mathrm{P}$, pelo método descrito por Raij et al. (1987). $\mathrm{O} \mathrm{pH}$ foi determinado em $\mathrm{CaCl}_{2}\left(0,01 \mathrm{~mol} \mathrm{~L}^{-1}\right)$. Foram realizadas determinações dos teores de $\mathrm{N}$, por microkjeldhal, e S, por turbidimetria do sulfato de bário, na parte aérea da forrageira na estação das águas e na estação seca.

Foi realizada a análise de variância, aplicandose os testes F e Tukey apenas para os tratamentos que receberam fertilização, a fim de se avaliar o efeito das doses de nutrientes estudadas e de suas interações. Foram realizadas regressões entre a produção de matéria seca e as doses de fertilizantes para melhor avaliação dos resultados.

\section{Resultados e Discussão}

A produção de matéria seca (Figura 1) em todos os tratamentos que receberam a calagem e a fertilização (completa + micronutrientes) foi superior à produção da pastagem degradada do tratamento testemunha nos dois anos, corroborando os resultados obtidos por Soares Filho et al. (1992) e Oliveira (2003). No primeiro ano, a pastagem degradada produziu $2,2 \mathrm{tha}^{-1}$ de MS, enquanto a produção média dos tratamentos que receberam calagem e fertilização foi de $7 \mathrm{tha}^{-1} \mathrm{de}$ MS; o mesmo ocorreu no segundo ano, quando a pastagem degradada produziu $6,3 \mathrm{t} \mathrm{ha}^{-1} \mathrm{de} \mathrm{MS}$, valor inferior às $11,8 \mathrm{t} \mathrm{ha}^{-1}$ produzidas em média pelos tratamentos com calagem e fertilização $(\mathrm{P} \leq 0,01)$. 
A resposta em produção de forragem às doses de nitrogênio nos dois anos foi linear (Figura 2), mas os tratamentos apresentaram diferentes respostas ao longo do tempo. No primeiro ano, não houve diferença para as doses de $\mathrm{N}$ e $\mathrm{N}+\mathrm{S}(\mathrm{P} \leq 0,05)$, enquanto, no segundo ano, o tratamento com $210 \mathrm{~kg} \mathrm{ha}^{-1} \mathrm{ano}^{-1} \mathrm{de}$ $\mathrm{N}$ e $230 \mathrm{~kg} \mathrm{ha}^{-1} \mathrm{ano}^{-1} \mathrm{de} \mathrm{S}$ proporcionou maior produção de forragem que os tratamentos com doses de 70 e $140 \mathrm{~kg} \mathrm{ha}^{-1} \mathrm{ano}^{-1}$ de $\mathrm{N}$, seja na presença ou na ausência de $\mathrm{S}$, e produção semelhante ao tratamento com $210 \mathrm{~kg} \mathrm{ha}^{-1}$ de $\mathrm{N}$ e sem S (Figura 1). As respostas em produção de forragem, em relação às doses de $\mathrm{N}$

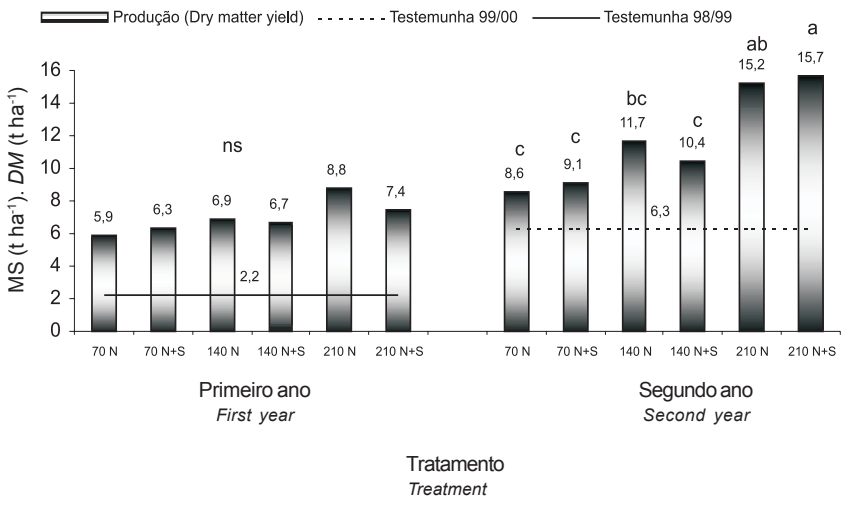

Figura 1 - Produção de matéria seca de forragem $\left(\mathrm{t} \mathrm{ha} \mathrm{a}^{-1}\right)$, em função de doses de nitrogênio e de enxofre em dois anos. Médias seguidas por letras distintas, no mesmo ano experimental, diferem $(P \leq 0,01)$ pelo teste Tukey.

Figure 1 - Dry matter yield ( $\left.t \mathrm{ha}^{-1}\right)$, in function of nitrogen and sulfur levels during two years. Means followed by different letter, within an experimental year, are different $(P \leq .01)$ by Tukey test.

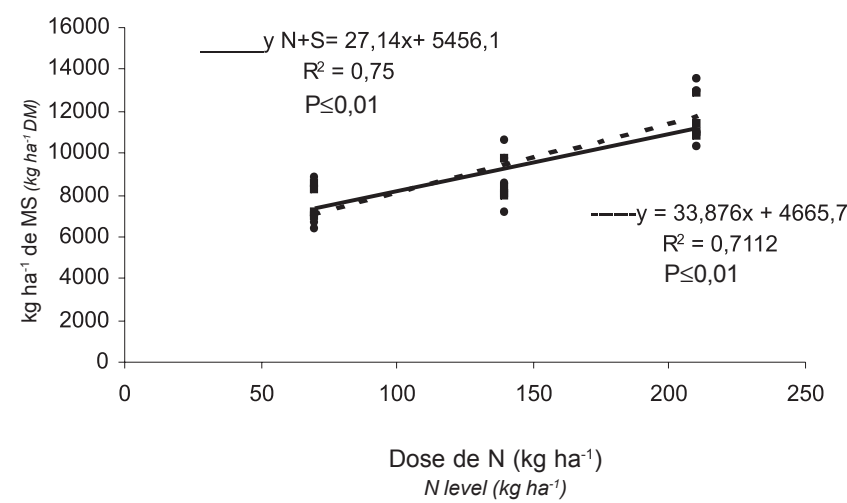

Figura 2 - Relação entre a produção de forragem e os níveis da fertilização nitrogenada em Brachiaria brizantha cv. Marandu.

Figure 2 - Relation between accumulated forage dry matter yield and nitrogen fertilizer levels in Brachiaria brizantha cv. Marandu. e $\mathrm{S}$, foram maiores no segundo que no primeiro ano (Figuras 1 e 3). Resultados semelhantes foram obtidos por outros autores (Oliveira et al., 2003; Cunha et al., 2001) e podem estar relacionados à necessidade de recuperação das estruturas da planta forrageira, como a coroa e o sistema radicular, para que a mesma possa expressar seu potencial de resposta em produtividade (Oliveira, 2001) e também a melhor eficiência do uso do nitrogênio pela pastagem (Oliveira \& Corsi, 2001). No primeiro ano, para cada unidade de nitrogênio aplicado, foram produzidos, na média de todos os tratamentos, $58 \mathrm{~kg}$ de MS da parte aérea de forragem, enquanto, no segundo ano, esse valor foi de $93 \mathrm{~kg}$ $(\mathrm{P} \leq 0,05)$. Este fato aponta para a necessidade de desenvolvimento de estudos de longa duração, sobretudo quando se tratar de nutrição de plantas forrageiras em estandes degradados e em recuperação.

A melhor eficiência do uso do nitrogênio no segundo ano foi acompanhada por maior extração e acúmulo de $\mathrm{N}$ na parte aérea da Brachiaria (Figura 4). No primeiro ano, apenas para os tratamentos com $70 \mathrm{~kg}$ ha $^{-1}$ ano $^{-1}$ de $\mathrm{N}$ o acúmulo de $\mathrm{N}$ na parte aérea foi superior à dose aplicada na fertilização. No segundo ano, em cada tratamento, o acúmulo de $\mathrm{N}$ na parte aérea foi maior que as doses aplicadas, evidenciando que o sistema de produção teve capacidade para

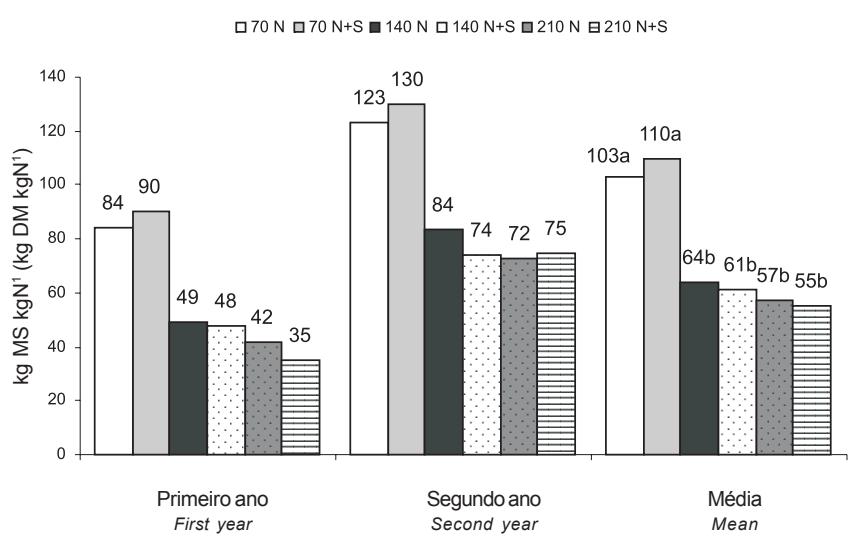

Figura 3 - Eficiência do uso de nitrogênio $\left(\mathrm{kgMS} \mathrm{kgN}^{-1}\right)$, em função de diferentes doses de $\mathrm{N}$ e $\mathrm{S}$ na produção de matéria seca de forragem em Brachiaria brizantha cv. Marandu. Médias seguidas por letras distintas, diferem pelo teste Tukey $(\mathrm{P} \leq 0,01)$.

Figure 3 - Nitrogen efficiency $\left(\mathrm{kgDM} \mathrm{kgN}^{-1}\right)$ in function of $\mathrm{N}$ and $S$ levels to the dry matter yield in Brachiaria decumbens cv. Marandu. Means followed by different letter are different $(P \leq 0.01)$ by Tukey test. 


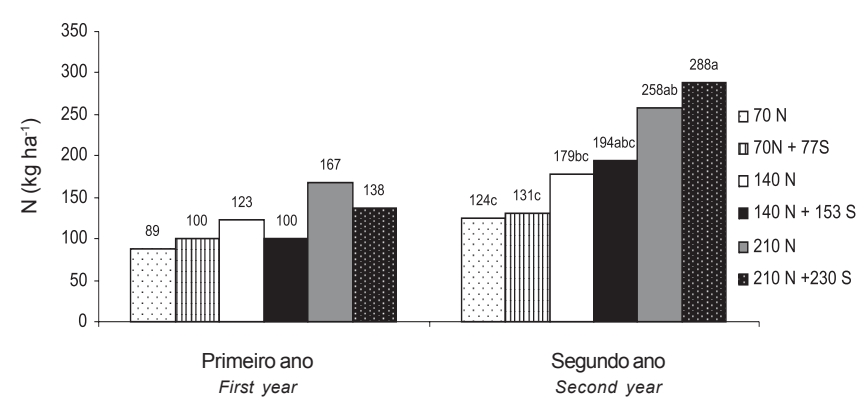

Figura 4 - Acúmulo de nitrogênio na parte aérea de Brachiaria brizantha cv. Marandu. Médias seguidas por letras distintas, no mesmo ano experimental, diferem $(P \leq 0,01)$ pelo teste Tukey.

Figure 4 - Nitrogen accumulation in the plant shoot of Brachiaria brizantha cv. Marandu. Means followed by different letter, within an experimental year, are different $(P \leq 0.01)$ by Tukey test.

suprir a exigência nutricional da planta. Os acúmulos de nitrogênio, superiores às doses de fertilizantes testadas, foram acompanhados de queda nos teores de matéria orgânica no solo (Figura 5). A pastagem extraiu e acumulou $\mathrm{N}$ mineralizado da matéria orgânica do solo, indicando que, provavelmente, o sistema de produção em pastagens foi capaz de suprir $\mathrm{N}$ para manter a produção utilizando a ciclagem de nutrientes, por meio da mineralização de matéria orgânica, até atingir um ponto de equilíbrio. A indicação desses pontos de equilíbrio para diferentes níveis de produção de matéria seca de forragem, por meio de curvas de reposta ao nitrogênio ao longo dos anos, seria de grande valia para o manejo da fertilização nitrogenada em pastagens, principalmente quando o sistema se encontra em recuperação.

Os teores de nitrogênio e a relação $\mathrm{N}$ :S na parte aérea da forragem foram maiores na estação das águas que na seca (Tabela 2). Na estação seca, o forte déficit hídrico afetou não só a produção, mas também a absorção do nitrogênio (Figura 6). Apenas para os tratamentos que receberam doses de $140 \mathrm{~kg} \mathrm{ha}^{-1} \mathrm{ano}^{-1} \mathrm{de}$ $\mathrm{N}+\mathrm{S}$ e $210 \mathrm{~kg} \mathrm{ha}^{-1} \mathrm{ano}^{-1}$ de $\mathrm{N}+\mathrm{S}$ os teores de enxofre foram maiores na época das águas, enquanto, para os outros tratamentos, os teores de enxofre foram semelhantes nas diferentes épocas do ano.

Os teores de enxofre e nitrogênio na forragem diferiram em função das diferentes doses de nitrogênio e enxofre (Tabela 2). Nos tratamentos sem enxofre, o teor deste na parte aérea ficou bastante próximo ou

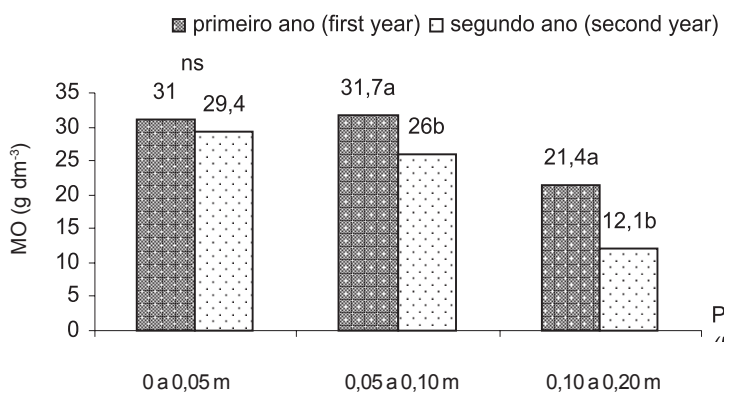

Figura 5 - Teor de matéria orgânica no solo, em função do tempo em diferentes profundidades. Médias seguidas por letras distintas, na mesma profundidade, diferem $(\mathrm{P} \leq 0,05)$ pelo teste Tukey.

Figure 5 - Soil organic matter content, in function of the time in different depths. Means followed by different letter, within a depth, are different $(P \leq 0.05)$ by Tukey test.

abaixo do teor mínimo $\left(0,8 \mathrm{~g} \mathrm{~kg}^{-1}\right)$ da faixa adequada do nutriente (Werner et al., 1996) para Brachiaria brizantha. Quando foram realizadas as fertilizações com enxofre, os valores foram mais altos, mas não ultrapassaram $1,7 \mathrm{~g} \mathrm{~kg}^{-1}$. O teor de $1,7 \mathrm{~g} \mathrm{~kg}^{-1}$, obtido com o nível de $253 \mathrm{~kg} \mathrm{ha}^{-1}$ de enxofre, é bastante inferior àquele que poderia causar problemas na nutrição animal de bovinos, de $4 \mathrm{~g} \mathrm{~kg}^{-1}$ (NRC, 1989).

Apesar do aumento na produção de forragem, que poderia diluir o efeito das doses de nitrogênio empregadas, os teores de nitrogênio na forragem durante a época das águas elevaram-se com o aumento das doses de nitrogênio aplicadas na fertilização (Tabela 2). Embora a literatura apresente resultados controversos, muitos autores obtiveram resultados semelhantes (Zanetti et al., 1992; Ruggieri et al., 1995; Correa et al., 1998), quando a forragem foi cortada em idade adequada, como neste experimento, em que se adotou período de descanso para B. brizantha de 35 dias.

A relação $\mathrm{N}: \mathrm{S}$ foi afetada pelo padrão no acúmulo de $\mathrm{N}$ e $\mathrm{S}$ na época das águas e da seca. $\mathrm{Na}$ época das águas, foi possível detectar diferenças entre os tratamentos e usar a relação como indicativo da nutrição da forrageira (Tabela 2). Os tratamentos que não receberam enxofre apresentaram relação $\mathrm{N}: \mathrm{S}$ muito alta e superior a relação de 16,5:1 apontada por Scott (1983), ou seja, houve falta de S no sistema, enquanto os tratamentos que receberam enxofre apresentaram relações inferiores a $16,8: 1$, adequadas à nutrição 


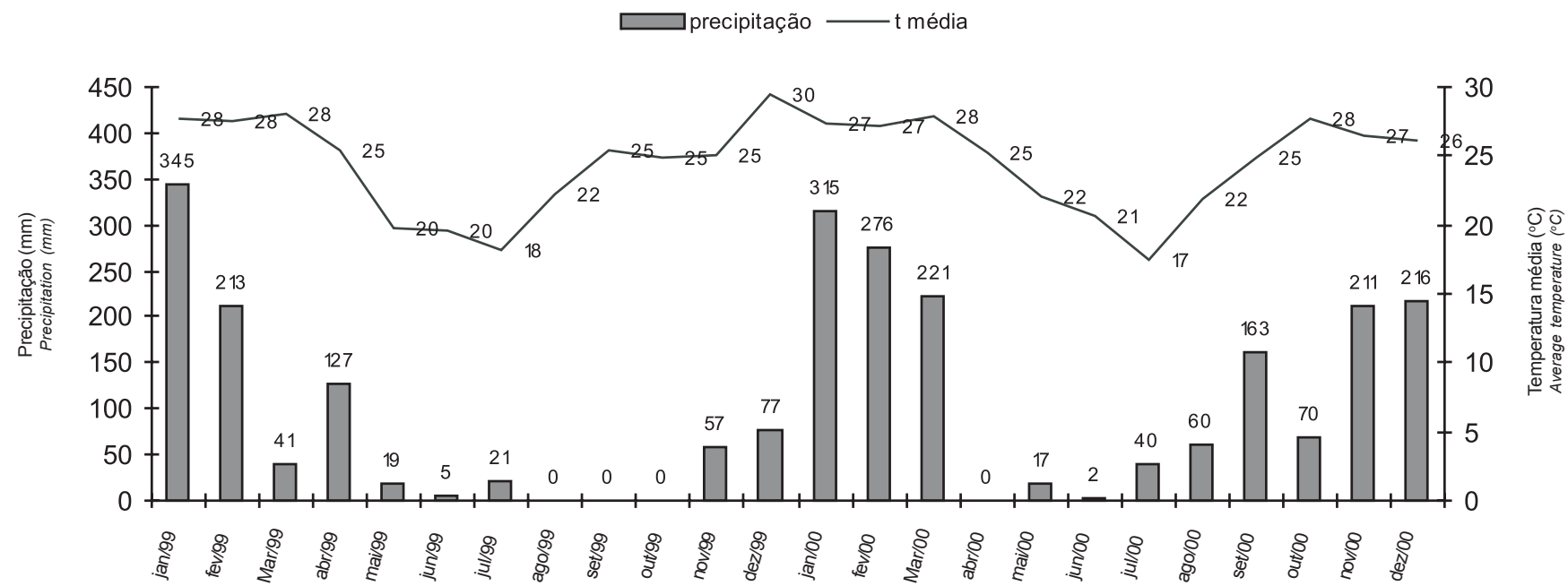

Figura 6 - Condições climatológicas na área experimental em Descalvado, SP.

Figure 6 - Climate conditions in the experimental field, Descalvado, SP.

Tabela 2 - Teores de nitrogênio e enxofre e a relação N:S na parte aérea de Brachiaria brizantha cv. Marandu em função de diferentes doses de N+S e da estação do ano

Table 2 - Nitrogen and sulfur contents and N:S ratio in plant shoot of Brachiaria brizantha cv. Marandu, in function of different N+S levels and year season

\begin{tabular}{|c|c|c|c|c|c|c|}
\hline \multirow{3}{*}{$\begin{array}{l}\text { Tratamento } \\
\text { Treatment }\end{array}$} & \multicolumn{2}{|c|}{$\mathrm{N}\left(\mathrm{g} \mathrm{N} \mathrm{kg} \mathrm{MS}^{-1}\right)^{1}$} & \multicolumn{2}{|c|}{$\mathrm{S}\left(\mathrm{g} \mathrm{S} \mathrm{kg} \mathrm{MS}^{-1}\right)$} & \multicolumn{2}{|c|}{ Relação N:S (N:S ratio) } \\
\hline & Águas & Seca & Águas & Seca & Águas & Seca \\
\hline & Rainy season & Dry season & Rainy season & Dry season & Rainy season & Dry season \\
\hline $70 \mathrm{~kg} \mathrm{~N}$ & $15,9 \mathrm{Ac}$ & 7,7 B a & $0,7 \mathrm{Ac}$ & $0,9 \mathrm{~A} \mathrm{bc}$ & $23,8 \mathrm{~A} \mathrm{ab}$ & $11,9 \mathrm{~B} \mathrm{ab}$ \\
\hline $140 \mathrm{~kg} \mathrm{~N}$ & $18,6 \mathrm{~A} \mathrm{ab}$ & $8,3 \mathrm{~B} \mathrm{a}$ & $0,8 \mathrm{~A} \mathrm{c}$ & $0,7 \mathrm{~A} \mathrm{c}$ & $28,1 \mathrm{~A} \mathrm{a}$ & $16,7 \mathrm{~B} \mathrm{a}$ \\
\hline $210 \mathrm{~kg} \mathrm{~N}$ & $19,5 \mathrm{~A} \mathrm{a}$ & $8,5 \mathrm{~B} \mathrm{a}$ & $0,8 \mathrm{~A} \mathrm{c}$ & $0,9 \mathrm{~A} \mathrm{bc}$ & $31,2 \mathrm{~A} \mathrm{a}$ & $12,6 \mathrm{~B} \mathrm{a}$ \\
\hline $70 \mathrm{~kg} \mathrm{~N}$ e $77 \mathrm{~kg} \mathrm{~S}$ & $16,4 \mathrm{~A} \mathrm{bc}$ & $7,9 \mathrm{~B} \mathrm{a}$ & $1,1 \mathrm{~A} \mathrm{bc}$ & $1,0 \mathrm{~A} \mathrm{bc}$ & $20,8 \mathrm{~A} \mathrm{ab}$ & $9,1 \mathrm{~B} \mathrm{ab}$ \\
\hline $140 \mathrm{~kg} \mathrm{~N} \mathrm{e} 153 \mathrm{~kg} \mathrm{~S}$ & $18,2 \mathrm{~A} a b$ & $8,3 \mathrm{~B} \mathrm{a}$ & $1,3 \mathrm{~A} \mathrm{~b}$ & $1,1 \mathrm{~B} \mathrm{ab}$ & $16,8 \mathrm{~A} \mathrm{~b}$ & $8,9 \mathrm{~B} \mathrm{~b}$ \\
\hline $210 \mathrm{~kg} \mathrm{~N} \mathrm{e} 230 \mathrm{~kg} \mathrm{~S}$ & $19,5 \mathrm{~A} \mathrm{a}$ & $8,1 \mathrm{~B} \mathrm{a}$ & $1,7 \mathrm{~A} \mathrm{a}$ & $1,4 \mathrm{~B} \mathrm{a}$ & $13,9 \mathrm{~A} \mathrm{~b}$ & $6,5 \mathrm{~B} \mathrm{~b}$ \\
\hline Testemunha & 18,9 & 9,3 & 1,1 & 0,9 & 17,8 & 10,3 \\
\hline
\end{tabular}

Médias para cada variável seguidas por letras maiúsculas distintas, na mesma linha, diferem $(\mathrm{P} \leq 0,01)$ pelo teste Tukey. Means followed by different uppercase letter, within a row, are different $(P \leq 0.01)$ by Tukey test.

Médias para cada variável seguidas por letras minúsculas distintas, na mesma coluna, diferem $(P \leq 0,01)$ pelo teste Tukey.

Means followed by different lowercase letter, within columns, are different $(P \leq 0.01)$ by Tukey test.

mineral da forrageira, denotando fornecimento suficiente de $\mathrm{S}$ em relação às doses de $\mathrm{N}$ usadas. Na época seca, como o teor de nitrogênio foi muito baixo, a relação $\mathrm{N}$ : S foi menor e o uso da relação como critério de diagnóstico tornou-se comprometida, uma vez que, apesar da baixa produção de forragem nesta época (Figura 1), a relação N:S estava adequada. Atualmente, outros critérios para diagnóstico da nutrição adequada de enxofre em forrageiras têm sido utilizados, como a relação $\mathrm{S}_{-} \mathrm{SO}_{4}: \mathrm{S}$-total (Chiy \& Phillips, 1998).

Nos tratamentos com aplicação de enxofre, a extração de S pela planta foi menor que as doses do nutriente aplicado ao solo (Figura 7). Mesmo quando se empregou a menor dose $\left(77 \mathrm{~kg} \mathrm{ha}^{-1} \mathrm{ano}^{-1}\right.$ de S), as exigências nutricionais da $B$. brizantha foram atendidas, indicando que a substituição da fonte nitrogenada de uréia pelo sulfato de amônio nas coberturas nitrogenadas pode ser parcial e menor que a realizada neste estudo. No primeiro ano, a aplicação de S dentro da mesma dose de $\mathrm{N}$ não influiu na quantidade de acúmulo de nutriente, ao passo que, no segundo ano, nas doses de 140 e $210 \mathrm{~kg} \mathrm{ha}^{-1} \mathrm{ano}^{-1}$ de $\mathrm{N}$ houve maior acúmulo de $\mathrm{S}$ com a aplicação de $\mathrm{S}$.

Os teores de fósforo na parte aérea da forragem não variaram em função das diferentes doses de $\mathrm{Ne} \mathrm{S}$ e épocas do ano, apresentando média de $1,56 \mathrm{~g} \mathrm{~kg}^{-1}$.

A quantidade de raízes, em todas as profundidades, tanto no primeiro quanto no segundo ano, e sua infecção 


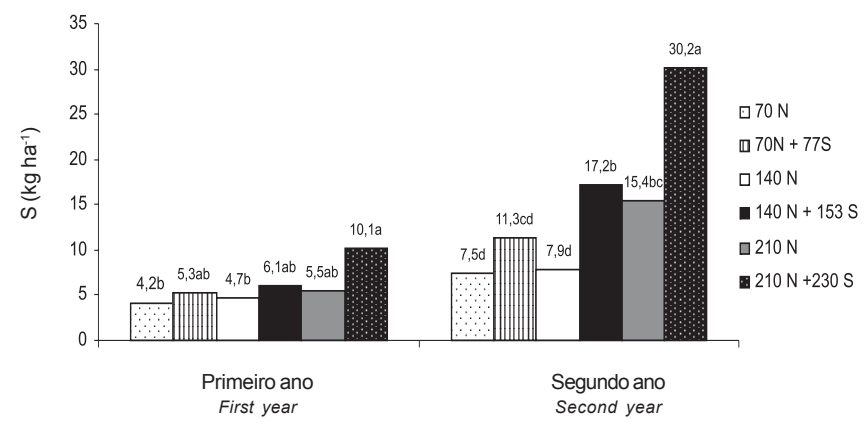

Figura 7 - Acúmulo de enxofre na parte aérea de Brachiaria brizantha cv. Marandu. Médias seguidas por letras distintas, no mesmo ano experimental, diferem $(P \leq 0,05)$ pelo teste Tukey.

Figure 7 - Sulfur accumulation in the plant shoot of Brachiaria brizantha cv. Marandu. Means followed by different letter, within an experimental year, are different $(P \leq 0.05)$ by Tukey test.

por micorrizas não diferiram com as diferentes doses de $\mathrm{N}$ e $\mathrm{S}$ avaliadas. Foram encontradas, em média, 4,$24 ; 4,42 ; 5,60$ toneladas ha $^{-1}$ de raízes para as profundidades de $0-0,05 ; 0,05-0,10$ e $0,10-0,30 \mathrm{~m}$, respectivamente. Na profundidade de $0,05-0,10 \mathrm{~m}$, a quantidade de raízes encontrada aumentou de 3,77 toneladas ha ${ }^{-1}$ para 5,46 toneladas ha-1 $(\mathrm{Pd} \leq 0,01)$ do primeiro para o segundo ano, como também foi observado para B. decumbens em recuperação (Oliveira et al., 2003).

Houve efeito do tempo para as diferentes características químicas do solo. A saturação por bases, os teores de $\mathrm{Ca}$ e de $\mathrm{Mg}$ na profundidade de 0-0,05 $\mathrm{m}$ e a CTC na camada de $0-0,10 \mathrm{~m}$ aumentaram do primeiro para o segundo ano (Tabela 3). Entretanto, a saturação por bases e os teores de $\mathrm{Ca}$ e $\mathrm{Mg}$ diminuíram nas profundidades de 0,05-0,10 m e 0,10-0,30 m, evidenciando a baixa mobilidade destes elementos no perfil do solo ocupado por pastagens que receberam calagem superficial. A aplicação de altas doses de fertilizantes não foram suficientes para auxiliar no carreamento de $\mathrm{Ca}$ e $\mathrm{Mg}$, fato também observado por Oliveira et al. (2003) e Allen \& Hossner (1991). Após dois anos de calagem consecutiva, em que se almejava atingir saturação por bases de $70 \%$, o valor médio não ultrapassou $48,6 \%$ na camada de $0-0,05 \mathrm{~m}$, como ocorrido e discutido em vários outros trabalhos (Oliveira et al., 2003; Caires \& Fonseca, 2000; Guimarães, 2000).

Os teores de fósforo aumentaram na camada até $0,10 \mathrm{~m}$, mas diminuíram na camada de 0,10 a $0,30 \mathrm{~m}$

Tabela 3 - Teores de nutrientes no solo em diferentes profundidades

Table 3 - Soil nutrientes content in different depths

\begin{tabular}{|c|c|c|c|}
\hline $\begin{array}{l}\text { Variável } \\
\text { Variable }\end{array}$ & $\begin{array}{l}\text { Profundidade } \\
\text { Depth }\end{array}$ & $\begin{array}{l}\text { Primeiro ano } \\
\text { First year }\end{array}$ & $\begin{array}{l}\text { Segundo ano } \\
\text { Second year }\end{array}$ \\
\hline $\mathrm{Ca}^{++}\left(\mathrm{mmol}_{\mathrm{c}} \mathrm{dm}^{-3}\right)$ & $\begin{array}{c}0-0,05 \mathrm{~m} \\
0,05-0,10 \mathrm{~m} \\
0,10-0,30 \mathrm{~m}\end{array}$ & $\begin{array}{r}11,2 \mathrm{~b} \\
9,7 \mathrm{a} \\
4,4 \mathrm{a} \\
\end{array}$ & $\begin{array}{r}15,9 \mathrm{a} \\
7,5 \mathrm{~b} \\
3,3 \mathrm{~b} \\
\end{array}$ \\
\hline $\mathrm{Mg}^{++}\left(\mathrm{mmol}_{\mathrm{c}} \mathrm{dm}^{-3}\right)$ & $\begin{array}{c}0-0,05 \mathrm{~m} \\
0,05-0,10 \mathrm{~m} \\
0,10-0,30 \mathrm{~m}\end{array}$ & $\begin{array}{r}7,0 \mathrm{~b} \\
6,2 \mathrm{~ns} \\
3,1 \mathrm{a}\end{array}$ & $\begin{array}{c}10,5 \mathrm{a} \\
4,9 \mathrm{~ns} \\
2,0 \mathrm{~b}\end{array}$ \\
\hline $\mathrm{K}^{+}\left(\mathrm{mmol}_{\mathrm{c}} \mathrm{dm}^{-3}\right)$ & $\begin{array}{c}0-0,05 \mathrm{~m} \\
0,05-0,10 \mathrm{~m} \\
0,10-0,30 \mathrm{~m} \\
\end{array}$ & $\begin{array}{l}1,1 \mathrm{a} \\
0,8 \mathrm{a} \\
0,4 \mathrm{a} \\
\end{array}$ & $\begin{array}{l}0,7 \mathrm{~b} \\
0,6 \mathrm{~b} \\
0,3 \mathrm{~b} \\
\end{array}$ \\
\hline $\mathrm{CTC}\left(\mathrm{mmol}_{\mathrm{c}} \mathrm{dm}^{-3}\right)$ & $\begin{array}{c}0-0,05 \mathrm{~m} \\
0,05-0,10 \mathrm{~m} \\
0,10-0,30 \mathrm{~m}\end{array}$ & $\begin{array}{l}47,3 \mathrm{~b} \\
49,1 \mathrm{~b} \\
37,5 \mathrm{~ns}\end{array}$ & $\begin{array}{c}56,9 \mathrm{a} \\
58,5 \mathrm{a} \\
41,3 \mathrm{~ns}\end{array}$ \\
\hline Saturação por bases (\%) & $\begin{array}{c}0-0,05 \mathrm{~m} \\
0,05-0,10 \mathrm{~m} \\
0,10-0,30 \mathrm{~m}\end{array}$ & $\begin{array}{l}41,0 \mathrm{~b} \\
35,2 \mathrm{a} \\
21,2 \mathrm{a}\end{array}$ & $\begin{array}{l}48,6 \mathrm{a} \\
22,1 \mathrm{~b} \\
14,3 \mathrm{~b}\end{array}$ \\
\hline $\mathrm{P}\left(\mathrm{mg} \mathrm{dm}^{-3}\right)$ & $\begin{array}{c}0-0,05 \mathrm{~m} \\
0,05-0,10 \mathrm{~m} \\
0,10-0,30 \mathrm{~m}\end{array}$ & $\begin{array}{l}5,8 \mathrm{~b} \\
3,2 \mathrm{~b} \\
6,8 \mathrm{a}\end{array}$ & $\begin{array}{l}9,6 \mathrm{a} \\
5,8 \mathrm{a} \\
3,0 \mathrm{~b}\end{array}$ \\
\hline $\mathrm{S}\left(\mathrm{mg} \mathrm{dm}^{-3}\right)$ & $\begin{array}{c}0-0,05 \mathrm{~m} \\
0,05-0,10 \mathrm{~m} \\
0,10-0,30 \mathrm{~m}\end{array}$ & $\begin{array}{c}7,7 \mathrm{~ns} \\
8,1 \mathrm{~b} \\
11,2 \mathrm{~ns} \\
\end{array}$ & $\begin{array}{r}7,8 \mathrm{~ns} \\
12,0 \mathrm{a} \\
10,0 \mathrm{~ns} \\
\end{array}$ \\
\hline
\end{tabular}

Médias para cada variável seguidas por letras distintas, na mesma linha, diferem $(P \leq 0,05)$ pelo teste Tukey. Means followed by different letter, within a row, differ $(P \leq .05)$ by Tukey test. 
(Tabela 3), o que está relacionado à baixa mobilidade do elemento no solo (Raij, 1991). A elevação dos teores na camada superficial indica que a dose de $80 \mathrm{~kg} \mathrm{ha}^{-1}$ ano $^{-1}$ de $\mathrm{P}_{2} \mathrm{O}_{5}$ foi suficiente para elevar gradativamente os níveis de $\mathrm{P}$ no solo da pastagem.

Os teores de potássio diminuíram em todas as profundidades estudadas do primeiro para o segundo ano (Tabela 3). Portanto, o critério de elevar a saturação de potássio a 5\% na CTC (Corsi \& Nussio, 1994) não foi suficiente para proporcionar aumento nos teores de $\mathrm{K}$ no solo, levando à inferência de que curvas de calibração da fertilização com potássio para pastagens degradadas também são necessárias.

$\mathrm{O}$ enxofre acumulou nas camadas mais profundas do solo, de modo que, na camada de 0,05-0,10 m, houve aumentos nos teores de $\mathrm{S}$ no solo do primeiro para o segundo ano (Tabela 2). Cantarella (1996) explica que o sulfato é mais retido em camadas subsuperficiais com reação ácida, em virtude da presença de cargas positivas e menores teores de ânions, como o fosfato, que competem por sítios de adsorção. $\mathrm{Na}$ Tabela 3 observa-se que os teores médios de $\mathrm{P}$ e a saturação por bases foram baixos, enquanto a acidez foi alta em profundidade, fatos que justificam o acúmulo de S. Nguyen \& Goh (1993) relataram este comportamento do enxofre e enfatizaram a ocorrência de possíveis perdas por lixiviação advindas desse processo. Em função disto, as diferenças entre os tratamentos foram verificadas apenas na camada de

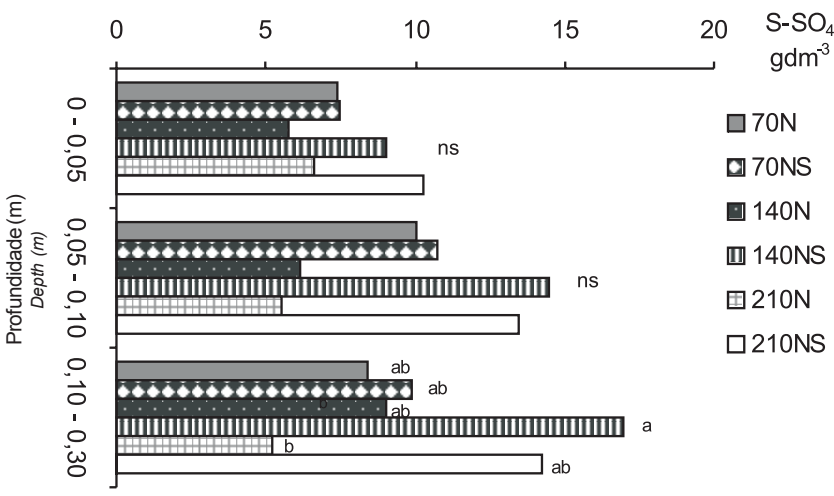

Figura 8 - Teores de S no solo em diferentes profundidades. Médias seguidas por letras distintas, na mesma profundidade, diferem $(\mathrm{P} \leq 0,05)$ pelo teste Tukey.

Figure 8 - $S$ soil content in different depth. Means followed by different letter, within a depth, are different $(P \leq 0.05)$ by Tukey test.
0,10-0,30 m no solo; os teores de enxofre no solo do tratamento que recebeu $140 \mathrm{~kg} \mathrm{~N}+\mathrm{S}$ foram superiores aos do tratamento que recebeu $210 \mathrm{~kg} \mathrm{ha}^{-1} \mathrm{ano}^{-1} \mathrm{de} \mathrm{N}$, enquanto os dos demais foram intermediários, mas não diferentes estatisticamente (Figura 8).

\section{Conclusões}

A calagem e as fertilizações, associadas ao manejo adequado da planta forrageira, promoveram a recuperação da pastagem, representada pelo aumento em produtividade.

A reposta em produção de forragem foi linear em função das diferentes doses de $\mathrm{N}$, tanto na presença quanto na ausência de enxofre.

A eficiência do uso do nitrogênio foi maior no segundo ano.

A extração de nitrogênio foi maior e a de enxofre menor que as doses empregadas. Curvas de resposta ao uso de enxofre devem contemplar menores doses que $70 \mathrm{~kg} \mathrm{ha}^{-1}$ ano $^{-1}$ de $\mathrm{N}$, mesmo para neossolos.

Houve queda no teor de matéria orgânica no solo em função do tempo.

Nos tratamentos que não receberam enxofre na fertilização, houve deficiência na forragem.

Apenas na época das águas, a relação N:S mostrou-se efetiva como critério para diagnóstico da adequada nutrição com $\mathrm{S}$ pela forrageira. $\mathrm{Na}$ estação seca, as relações são alteradas, em razão dos diferentes comportamentos de acúmulo de $\mathrm{N}$ e $\mathrm{S}$ na forrageira.

$\mathrm{Na}$ presença de fertilização sulfatada, houve acúmulo de $\mathrm{S}$ no solo nas camadas subsuperficiais.

\section{Literatura Citada}

ALLEN, E.R.; HOSSNER, L.R. Factors affecting the accumulation of surface-applied agricultural limestone in permanent pastures. Soil Science, v.151, p.240-248, 1991.

ANCHÃO, P.P. Algumas considerações sobre a recuperação de pastos degradados. Piracicaba: Fundação de Estudos Agrários Luiz de Queiroz, 1997. p.1-4. (Boletim do Leite, 39).

BODDEY, R.M.; ALVES, B.J.R.; OLIVEIRA, O.C. et al. A degradação das pastagens e o ciclo do nitrogênio. In: WORKSHOP: NITROGÊNIO NA SUSTENTABILIDADE DE SISTEMAS INTENSIVOS DE PRODUÇÃO PECUÁRIA, 2000, Dourados. Anais... Dourados: EMBRAPA, 2000. p.110-123.

CAIRES, E.F.; FONSECA, A.F. Adsorção de nutrientes pela soja cultivada no sistema de plantio direto em função da calagem na superfície. Bragantia, v.59, n.2, p.213-220, 2000.

CAMARGO, O.A.; MONIZ, A.C.; JORGE, L.A. et al. Métodos de análise química, mineralógica e física do Instituto Agronômico de Campinas. Campinas: Instituto Agronômico, 1986. 94p. (Boletim Técnico, 106). 
CANTARElla, H. Adubação com nitrogênio, potássio e enxofre. Campinas: Instituto Agronômico, 1996. p.22-26. (Boletim Técnico, 100)

CHIY, P.C.; PHILLIPS, C.J.C. Sodium fertilizer application to pasture. 6. Effects of combined applications with sulphur on herbage production and chemical composition in the season of application. Grass and Forage Science, v.53, p.1-10, 1998

CORSI, M; NUSSIO, L.G. Manejo do capim-elefante: correção e adubação do solo. In: SIMPÓSIO SOBRE MANEJO DA PASTAGEM, 10., 1994, Piracicaba. Anais... Piracicaba: Fundação de Estudos Agrários "Luiz de Queiroz”, 1994. p.87-111.

CORSI, M.; MARTHA JR., G.B. Manutenção da fertilidade do solo em sistemas intensivos de pastejo rotacionado In: SIMPÓSIO SOBRE MANEJO DA PASTAGEM, 14., 1997, Piracicaba. Anais... Piracicaba: Fundação de Estudos Agrários “Luiz de Queiroz”, 1997. p.161-193.

CORRÊA, L.A.; FREITAS, A.R.; BATISTA, L.A.R. Níveis de nitrogênio e freqüências de corte em 12 gramíneas forrageiras tropicais II. Qualidade da forragem. In: REUNIÃO ANUAL DA SOCIEDADE BRASILEIRA DE ZOOTECNIA, 35. 1998, Botucatu. Anais... Botucatu: Sociedade Brasileira de Zootecnia, 1198. p.518-520.

CUNHA, M.K.; SIEWERDT, L.; SILVEIRA JR., P. et al. Doses de nitrogênio e enxofre na produção e qualidade da forragem de campo natural de planossolo no Rio Grande do Sul. Revista Brasileira de Zootecnia, v.30, n.3, p.651-658, 2001.

GUIMARÃES, G.F.P.B. Avaliação de quatro forrageiras tropicais cultivada em dois solos da ilha de Marajó-PA submetidos a crescentes saturações por bases. Piracicaba: Escola Superior de Agricultura "Luiz de Queiroz", 2000. 197p. Dissertação (Mestrado em Ciência Animal e Pastagens) - Escola Superior de Agricultura “Luiz de Queiroz” 2000.

NGUYEN, M.L.; GOH, K.M. Sulphur cycling and its implications on sulphur fertilizer requirements of grazed grassland ecosystems. Agriculture, Ecosystems and Environment, v.49, p.173-206, 1994.

NATIONAL RESEARCH COUNCIL - NRC. Nutrient requirements of dairy cattle. Washington, D.C.: 1989. 157p.

OLIVEIRA, P.P.A.; OLIVEIRA,W.S.O.; CORSI, M. Estudo sobre recuperação de pastagens através de fertilização e mecanização. Piracicaba: Fundação de Estudos Agrários "Luiz de Queiroz", 2000. 36p. (Relatório Técnico)

OLIVEIRA, P.P.A.; CORSI, M. Eficiência da fertilização nitrogenada e sulfatada em pastagens. In: WORKSHOP SOBRE MANEJO DE FERTILIZANTES NITROGENADOS E SULFATADOS NA AGRICULTURA, 2001, Piracicaba. Anais... Piracicaba: 2001. 36p. CD-ROM.

OLIVEIRA, P.P.A. Manejo da calagem e da fertilização nitrogenada na recuperação de pastagens degradadas de Brachiaria sp. em solos arenosos. Piracicaba: Centro de Energia Nuclear na Agricultura, 2001. 110p. Tese (Doutorado em Ciências) - Centro de Energia Nuclear na Agricultura/ Universidade de São Paulo, 2001.

OLIVEIRA, P.P.A.; BOARETTO, A.E.; TRIVELIN, P.C.O. et al. Liming and fertilization for restoring degraded Brachiaria decumbens pasture on sandy soil. Scientia Agricola, v.60, n.1, p.125-131, 2003.
OLIVEIRA, P.P.A; TRIVELIN, P.C.O.; OLIVEIRA, W.S. et al. Eficiência da fertilização nitrogenada com uréia $\left({ }^{15} \mathrm{~N}\right)$ em Brachiaria brizantha cv. Marandu associada ao parcelamento de superfosfato simples e cloreto de potássio. Revista Brasileira de Ciência do Solo, v.27, p.613-620, 2003.

RAIJ, B.V. Fertilidade do solo e adubação. Piracicaba: CERES, POTAFOS, 1991. 343p.

RUGGIERI, A.C.; FAVORETTO, V.; MALHEIROS, E.B. Efeito de níveis de nitrogênio e regimes de corte na distribuição, na composição bromatológica e na digestibilidade "in vitro" da matéria seca da Brachiaria brizantha (Hochst) Stapf cv. Marandu. Revista da Sociedade Brasileira de Zootecnia, v.24, n.2, p.222-232, 1995.

SANTOS, A.R.; MONTEIRO, F.A. Produção e perfilhamento de Brachiaria decumbens Stapf. em função de doses de enxofre. Scientia Agricola, v.56, p.689-692, 1999.

SCOTT, N.M.; WATSON, M.E.; CALDWELL, K.S. Response of grassland to the application of sulphur at two sites in northeast Scotland. Journal of the Food and Agriculture, v.34, p.357-361, 1983.

SOARES FILHO, C.V.; MONTEIRO, F.A.; CORSI, M. Recuperação de pastagens degradadas. 1. Efeito de diferentes tratamentos de fertilização e manejo. Pasturas Tropicales, v.14, n.2, p.2-6, 1992

STOUT, W.L. Evaluating the "added nitrogen interaction" effect in forage grasses. Communications Soil Science Plant Analysis, v.26, p.2829-41, 1995.

VITTI, G.C.; NOVAES, N.J. Adubação com enxofre. In: SIMPÓSIO SOBRE CALAGEM E ADUBAÇÃO DE PASTAGENS, 1., 1985, Nova Odessa. Anais... Piracicaba: POTAFOS, 1986. p.191-231.

ZANETTI, M.A.; GOMIDE, C.A.; HERLING, V.R. et al. Influência de níveis de adubação nitrogenada e potássica e estágios de crescimento sobre o capim setária (Setaria anceps Stapf Ex Massey cv. Kazungula). II. Composição química. Revista da Sociedade Brasileira de Zootecnia, v.21, n.4, p.545-53, 1992.

ZIMMER, A.H.; MACEDO, M.C.M.; BARCELLOS, A.O. et al. Estabelecimento e recuperação de Brachiaria. In: SIMPÓSIO SOBRE MANEJO DA PASTAGEM, 11., 1994, Piracicaba. Anais... Piracicaba: Fundação de Estudos Agrários "Luiz de Queiroz", 1994. p.153-208.

WERNER, J.C.; PAULINO, V.T.; CANTARELLA, H. Recomendação de adubação e calagem para forrageiras. In: van RAIJ, B.; SILVA, N.M.; BATAGLIA, O.C. et al. (Eds.) Recomendação de adubação e calagem para o Estado de São Paulo. Campinas: Instituto Agronômico; Fundação IAC, 1996. p.263-271. 